\title{
VALUATION THEORY OF EXPONENTIAL HARDY FIELDS II: PRINCIPAL PARTS OF GERMS IN THE HARDY FIELD OF O-MINIMAL EXPONENTIAL EXPANSIONS OF THE REALS
}

\author{
FRANZ-VIKTOR KUHLMANN AND SALMA KUHLMANN
}

\begin{abstract}
We present a general structure theorem for the Hardy field of an o-minimal expansion of the reals by restricted analytic functions and an unrestricted exponential. We proceed to analyze its residue fields with respect to arbitrary convex valuations, and deduce a power series expansion of exponential germs. We apply these results to cast "Hardy's conjecture" (see [7, p.111]) in a more general framework. This paper is a follow up to [6] and is partially based on unpublished results of [4. A previous version [5] (which was dedicated to Murray A. Marshall on his 60th birthday) remained unpublished. In [9] our structure theorem for the residue fields was rediscovered and applied to the diophantine context. Due to this revived interest, we decided to rework the arXiv preprint [5] and submit it to the Marshall Memorial Volume.
\end{abstract}

\section{INTRODUCTION}

In this paper, we analyze the structure of the Hardy fields associated with o-minimal expansions of the reals with exponential function. More precisely, we take $T$ to be the theory of a polynomially bounded o-minimal expansion $\mathcal{P}$ of the ordered field of real numbers by a set $\mathcal{F}_{T}$ of real-valued functions. We assume that the language of $T$ contains a symbol for every 0-definable function, and that $T$ defines the restricted exponential and logarithmic functions. Now let $T(\exp )$ denote the theory of the expansion $(\mathcal{P}, \exp )$ where exp is the un-restricted real exponential function. Then also $T(\exp )$ is o-minimal, and admits quantifier elimination and a universal axiomatization in the language

Date: 12. 9. 2016.

2000 Mathematics Subject Classification. Primary: 06A05, 12J10, 12J15, 12L12, 13A18; Secondary: 03C60, 12F05, 12F10, 12F20.

Key words and phrases. polynomially bounded o-minimal expansion of the reals, real exponential function, convex valuation, value group, residue field, Hardy field, power series expansion. 
augmented by $\log [2$. We consider the Hardy field $H(\mathcal{P}$, exp) (see Section 2.2 for the definition). Our general assumptions (see Section 2.3) imply that $H(\mathcal{P}, \exp )$ is a model of $T(\exp )$ and is equal to the closure $L E_{\mathcal{F}_{T}}(x)$ of its subfield $\mathbb{R}(x)$ under real closure, $\mathcal{F}_{T}$, exp and its inverse log; here, $x$ denotes the germ of the identity function [1].

We study convex valuations on $H\left(\mathcal{P}\right.$, exp). To this end, for $\mathcal{F} \subseteq \mathcal{F}_{T}$, we introduce an intrinsic form of power series expansions for the elements of $L E_{\mathcal{F}}(x)$. We use monomials, which are the elements in the image of a suitable cross-section, together with coefficients from residue fields $L E_{\mathcal{F}}(x) w$ with respect to significant convex valuations $w$. We apply our results in particular to $\mathcal{F}=\mathcal{F}_{\text {an }}$ (the family of restricted analytic functions), $T=T_{\text {an }}$ (the polynomially bounded o-minimal theory of the expansion $\mathbb{R}_{\mathrm{an}}$ of the reals by restricted analytic functions), see [1] for more details about this theory.

The paper is organised as follows. In Section 2 we gather in a concise manner the necessary background. In Section 3 we prove our structure theorem for $L E_{\mathcal{F}}(x)$ and its residue fields, see Theorem 3.2. The main result leading to the definition of principal parts (see the definition in Section 4) is Theorem 4.1. Section 4 is dedicated to its proof. The final Section 5 considers applications to the Hardy field $H\left(\mathbb{R}_{\mathrm{an}, \exp }\right)$. The principal part of a function $h \in H\left(\mathbb{R}_{\mathrm{an}, \exp }\right)$ carries information about the asymptotic behavior of the function $\exp h(x)$ (Theorem 5.1). Corollary 5.2 gives a powerful criterion - using principal parts - for an exponential germ to be asymptotic to a composition of semialgebraic functions, exp, log and restricted analytic functions. This puts the particular solution of the Hardy problem in a more general framework; see the computations following Corollary 5.2. Finally, we provide a further application to embeddings of Hardy fields into fields of generalized power series, see Corollary 5.3 .

\section{SOME PRELIMINARIES}

2.1. Valuations. If $(K, w)$ is a valued field, then we write wa for the value of $a \in K$ and $w K$ for its value group $\{w a \mid 0 \neq a \in K\}$. Further, we write $a w$ for the residue of $a$, and $K w$ for the residue field. The valuation ring is denoted by $\mathcal{O}_{w}$. For generalities on valuation theory, see [8], and for convex valuations in particular see [7] or [3].

A valuation $w$ on an ordered field $K$ is called convex if $\mathcal{O}_{w}$ is convex. The convex valuation rings of an ordered field are linearly ordered by inclusion. If $\mathcal{O}_{w} \subsetneq \mathcal{O}_{w^{\prime}}$ then $w$ is said to be finer than $w^{\prime}$, and $w^{\prime}$ is a

coarsening of $w$. If $w$ and $w^{\prime}$ are two convex valuations on the same 
ordered field, we will write $w<w^{\prime}$ if $w$ is a proper coarsening of $w^{\prime}$, that is, if $\mathcal{O}_{w^{\prime}} \subsetneq \mathcal{O}_{w}$.

There is always a finest convex valuation, called the natural valuation. It is characterized by the fact that its residue field is archimedean. A valuation $w$ on an ordered field is convex if and only if the natural valuation is finer or equal to $w$. Throughout this paper, $v$ will always denote the natural valuation, unless stated otherwise.

If $a, b$ are elements of an ordered group or an ordered field, then we write $a \ll b<0$ if $a<b<0$ and $\forall n \in \mathbb{N}: a<n b$. Similarly, $a \gg b>0$ if $a>b>0$ and $\forall n \in \mathbb{N}: a>n b$. We set $|a|:=\max \{a,-a\}$. Then the natural valuation is characterized by:

$$
v a<v b \Leftrightarrow|a| \gg|b| \text {. }
$$

Note that if $\mathbb{R} \subset K$ and $a \in K$ with $v a=0$, then there is some $r \in \mathbb{R}$ such that $v(a-r)>0$. Further, $w r=0$ for every $r \in \mathbb{R}$ and every convex valuation $w$.

Lemma 2.1. Let $v, w$ be arbitrary valuations on some field $K$. Suppose that $v$ is finer than $w$. Then for all $a, b \in K$,

$$
v a \leq v b \Rightarrow w a \leq w b .
$$

In particular, wa $>0 \Rightarrow v a>0$. Further, $H_{w}:=\{v z \mid z \in K \wedge w z=0\}$ is a convex subgroup of the value group $v K$ of $v$. We have that $v z \in$ $H_{w} \Leftrightarrow z \in \mathcal{O}_{w}^{\times}$. There is a canonical isomorphism $w K \simeq v K / H_{w}$. Conversely, every convex subgroup of $v K$ is of the form $H_{w}$ for some valuation $w$ such that $v$ is finer or equal to $w$.

The valuation $v$ of $K$ induces a valuation $v / w$ on $K w$. There are canonical isomorphisms $v / w(K w) \simeq H_{w}$ and $(K w) v / w \simeq K v$. If $K w$ is embedded in $\mathcal{O}_{w}$ such that the restriction of the residue map is the identity on $K w$, then $v / w=\left.v\right|_{K w}$ (up to equivalence). Writing $v$ instead of $\left.v\right|_{K w}$, we then have that $v(K w)=H_{w}$ and $(K w) v=K v$.

We will call $H_{w}$ the convex subgroup associated with $w$ and $w$ the valuation associated with $H_{w}$. Since the isomorphism is canonical, we will write $w K=v K / H_{w}$.

The order type of the chain of nontrivial convex subgroups of an ordered abelian group $G$ is called the rank of $G$. If finite, then the rank is not bigger than the maximal number of rationally independent elements in $G$ (which is the dimension of its divisible hull as a $\mathbb{Q}$-vector space). In particular, $G$ has finite rank if it is finitely generated.

From (1) and (2) it follows that for every convex valuation $w$,

$$
|a| \leq|b| \Rightarrow w a \geq w b \text {. }
$$


Take any valued field $(K, v)$. A field of representatives for the residue field of $(K, v)$ is a subfield $k$ of $K$ such that $v$ is trivial on $k$ (or equivalently, $k$ is contained in the valuation ring $\mathcal{O}$ ), and for every $a \in \mathcal{O}$ there is $b \in k$ such that $v(a-b)>0$. It then follows that the residue map $\mathcal{O} \ni a \mapsto a v$ induces an isomorphism from $k$ to the residue field. A cross-section of $(K, v)$ is an embedding $\iota$ of the value group $v K$ in the multiplicative group $K^{\times}$such that $v \iota(\alpha)=\alpha$ for all $\alpha \in v K$.

2.2. Hardy fields. Let us recall some basic facts about Hardy fields (see Chapter 6, Section 2 in [7]). Assume that $T$ is the theory of any o-minimal expansion $\mathcal{R}$ of the ordered field of real numbers by realvalued functions. The Hardy field of $\mathcal{R}$, denoted by $H(\mathcal{R})$, is the set of germs at $\infty$ of unary $\mathcal{R}$-definable functions $f: \mathbb{R} \rightarrow \mathbb{R}$. Then $H(\mathcal{R})$ is an ordered differential field which contains $\mathcal{R}$ as a substructure. Let $x \in H(\mathcal{R})$ be the germ of the identity function. Then $H(\mathcal{R})$ is the closure of $\mathbb{R}(x)$ under all 0-definable functions of $\mathcal{R},[1$.

If $f, g$ are non-zero unary $\mathcal{R}$-definable functions on $\mathcal{R}$, then we will denote their germs in $H(\mathcal{R})$ by the same letters. The following holds for non-zero germs:

$$
v f=v g \Longleftrightarrow \lim _{x \rightarrow \infty} \frac{f(x)}{g(x)} \text { is a non-zero constant in } \mathbb{R} \text {. }
$$

The non-zero germs $f$ and $g$ are asymptotic if and only if this constant is 1 , and we have:

$$
f \text { and } g \text { are asymptotic } \Longleftrightarrow v(f-g)>v(g) \text {. }
$$

$$
\text { see [7, Lemma 6.22] }
$$

2.3. General assumptions on $T$. Throughout this paper, we will assume that $T$ is the theory of a polynomially bounded o-minimal expansion $\mathcal{P}$ of the ordered field of real numbers by real-valued functions. Further, we assume that $T$ defines the restricted exp and log. Then also $T(\exp )$ is o-minimal (cf. [2]). Here, $T(\exp )$ denotes the theory of the expansion $(\mathcal{P}$, exp) where exp is the un-restricted real exponential function.

We let $\mathcal{F}_{T}$ denote the set of function symbols in the language of $T$ and assume that there is a function symbol in $\mathcal{F}_{T}$ for each 0 -definable function of $\mathcal{P}$. This implies that $T$ admits quantifier elimination and a universal axiomatization. We let $\mathcal{F}$ denote any subset of $\mathcal{F}_{T}$.

We denote by $M$ a model of $T$. Often, we will assume further that $M$ is a model of $T$ (exp) (but will not distinguish notationally between $M$ and its reduct to the language of $T$.) Suppose that the field $K$ is a submodel (and hence elementary submodel) of $M$. Take $x_{i} \in$ 
$M, i \in I$. By $K\left\langle x_{i} \mid i \in I\right\rangle$ we denote the 0-definable closure of $K \cup\left\{x_{i} \mid i \in I\right\}$ in $M$. By our assumption on the language of $T$, it is the closure of $K \cup\left\{x_{i} \mid i \in I\right\}$ under $\mathcal{F}_{T}$, that is, the smallest subfield of $M$ containing $K \cup\left\{x_{i} \mid i \in I\right\}$ and closed under all functions which interpret the function symbols of $\mathcal{F}_{T}$ in $M$. Since $T$ admits a universal axiomatization and $K\left\langle x_{i} \mid i \in I\right\rangle$ is a substructure of $M$, it is a model of $T$. Since $T$ admits quantifier elimination, $K\left\langle x_{i} \mid i \in I\right\rangle$ is an elementary substructure of $M$.

For an arbitrary subfield $F \subseteq M$, the real closure $F^{\mathrm{r}}$ of $F$ can be taken to lie in $M$ since $M$ is real closed. We denote by $F^{\text {h }}$ the henselization of $(F, v)$. It can be taken to lie in $M$ since the natural valuation $v$ of the real closed field $M$ is henselian.

We let $F^{\mathcal{F}}$ denote the smallest subfield of $M$ which contains $F$ and is $\mathcal{F}$-closed, that is, closed under all functions on $M$ which are interpretations of function symbols in $\mathcal{F}$. Analogously, we define $F^{\mathrm{h} \mathcal{F}}$ to be the smallest subfield of $M$ which contains $F$ and is $\mathcal{F}$-closed and henselian w.r.t. $v$, and $F^{\mathrm{r} \mathcal{F}}$ to be the smallest such subfield which is in addition real closed. Note that $F^{\mathcal{F}} \subseteq F^{\mathrm{h} \mathcal{F}} \subseteq F^{\mathrm{r} \mathcal{F}}$.

\section{A general Structure theOrem For $L E_{\mathcal{F}}(x)$}

In what follows, we work under the assumptions of [7, Lemma 6.40; pp. 104-105]. More precisely, we let $M$ be a model of $T=T_{\text {an }}$ (or of $T_{\text {an }}(\exp )$, and $\mathcal{F} \subset \mathcal{F}_{\text {an }}$ be an arbitrary set of convergent power series representing restricted analytic functions, closed under partial derivatives, and containing the restricted exp and log.

For the proof Theorem 3.2 below, we need the following lemma.

Lemma 3.1. Let $M$ be a model of $T_{\mathrm{an}}, x_{i} \in M$ be such that the values $v x_{i}, i \in I$ are rationally independent. Further, let $w$ be any convex valuation. Assume that there is a subset $I_{w} \subset I$ such that $w x_{i}=0$ for all $i \in I_{w}$ and that the values $w x_{i}, i \in I \backslash I_{w}$ are rationally independent. Then

$w \mathbb{R}\left(x_{i} \mid i \in I\right)^{\mathrm{r} \mathcal{F}}=\bigoplus_{i \in I \backslash I_{w}} \mathbb{Q} w x_{i} \quad$ and $\quad w \mathbb{R}\left(x_{i} \mid i \in I\right)^{\mathrm{h} \mathcal{F}}=\bigoplus_{i \in I \backslash I_{w}} \mathbb{Z} w x_{i}$.

Further,

$$
\mathbb{R}\left(x_{i} \mid i \in I_{w}\right)^{\mathrm{r} \mathcal{F}}
$$

is a field of representatives for the residue field $\mathbb{R}\left(x_{i} \mid i \in I\right)^{\mathrm{r} \mathcal{F}} w$, and

$$
\mathbb{R}\left(x_{i} \mid i \in I_{w}\right)^{\mathrm{h} \mathcal{F}}
$$

is a field of representatives for the residue field $\mathbb{R}\left(x_{i} \mid i \in I\right)^{\mathrm{h} \mathcal{F}} w$. 
Assume in addition that all $x_{i}$ with $i \in I \backslash I_{w}$ are positive. Then the multiplicative group of $\mathbb{R}\left(x_{i} \mid i \in I \backslash I_{w}\right)^{\mathrm{r} \mathcal{F}}$ contains the divisible hull $\mathcal{X}$ of the group generated by all of these $x_{i}, \mathcal{X}$ is the image of a suitably chosen cross-section, and the following holds:

$$
\mathbb{R}\left(x_{i} \mid i \in I_{w}\right)^{\mathrm{r} \mathcal{F}}(\mathcal{X})^{\mathrm{r}}=\mathbb{R}\left(x_{i} \mid i \in I_{w}\right)^{\mathrm{r} \mathcal{F}}(\mathcal{X})^{\mathrm{h}} .
$$

Proof. The first part of this lemma is [7, Lemma 6.40].

Now assume that all $x_{i}>0$ for all $i \in I \backslash I_{w}$. Since $\mathbb{R}\left(x_{i} \mid i \in I \backslash I_{w}\right)^{\mathrm{r} \mathcal{F}}$ is real closed, it contains $x_{i}^{1 / k}$ for all $i \in I \backslash I_{w}$ and $k \in \mathbb{N}$. This yields that its multiplicative group contains the divisible hull $\mathcal{X}$ of the group generated by all of these $x_{i}$.

The restriction of $w$ to $\mathcal{X}$ is a group homomorphism onto the value group $w \mathbb{R}\left(x_{i} \mid i \in I\right)^{\mathrm{r} \mathcal{F}}$; it is injective since the values $w x_{i}, i \in I \backslash I_{w}$ are rationally independent. The inverse of this isomorphism is a crosssection with image $\mathcal{X}$.

From what we have proved, we obtain that $w \mathbb{R}\left(x_{i} \mid i \in I_{w}\right)^{\mathrm{r} \mathcal{F}}(\mathcal{X})^{\mathrm{h}}=$ $w \mathbb{R}\left(x_{i} \mid i \in I_{w}\right)^{\mathrm{r} \mathcal{F}}(\mathcal{X})=w \mathbb{R}\left(x_{i} \mid i \in I\right)^{\mathrm{r} \mathcal{F}}$, which is divisible. Further, the residue field of $\mathbb{R}\left(x_{i} \mid i \in I_{w}\right)^{\mathrm{r} \mathcal{F}}(\mathcal{X})^{\mathrm{h}}$ is $\mathbb{R}\left(x_{i} \mid i \in I_{w}\right)^{\mathrm{r} \mathcal{F}}$, which is real closed. Thus by [3, Theorem 4.3.7], $\mathbb{R}\left(x_{i} \mid i \in I_{w}\right)^{\mathrm{r} \mathcal{F}}(\mathcal{X})^{\mathrm{h}}$ is real closed, which gives equation (6) .

We now fix any non-archimedean model $M$ of $T(\exp )$ which contains $(\mathbb{R},+, \cdot,<, \mathcal{F}, \exp )$ as a substructure. We recall from the introduction that $L E_{\mathcal{F}}(x)$ denotes the closure of the subfield $\mathbb{R}(x)$ under real closure, $\mathcal{F}$, exp and its inverse log; here, $x$ denotes any infinitely large and positive element (i.e. $x>0$ and $v x<0$ ). The following is the structure theorem which we will put to work.

Theorem 3.2. $L E_{\mathcal{F}}(x)$ is of the form

$$
\mathbb{R}(\mathcal{X})^{\mathrm{r} \mathcal{F}}=\mathbb{R}(\mathcal{X})^{\mathrm{h} \mathcal{F}}
$$

where $\mathcal{X}$ is a subgroup of the multiplicative group of positive elements of $\operatorname{LE}_{\mathcal{F}}(x)$ which is the image of a cross-section, with the following properties:

a) $\mathcal{X}$ contains $x$ and $\log _{m} x$ for all $m \in \mathbb{N}$,

b) for every convex valuation $w$ on $L E_{\mathcal{F}}(x)$, if

$$
\mathcal{X}_{w}:=\left\{x^{\prime} \in \mathcal{X} \mid w x^{\prime}=0\right\}
$$

then

$$
\mathbb{R}\left(\mathcal{X}_{w}\right)^{\mathrm{r} \mathcal{F}}=\mathbb{R}\left(\mathcal{X}_{w}\right)^{\mathrm{h} \mathcal{F}} \subseteq L E_{\mathcal{F}}(x)
$$


is a field of representatives for the residue field $L E_{\mathcal{F}}(x) w$. Identifying $L E_{\mathcal{F}}(x) w$ with this field of representatives, we obtain that

$$
L E_{\mathcal{F}}(x) w \subseteq L E_{\mathcal{F}}(x) w^{\prime} \subseteq L E_{\mathcal{F}}(x)
$$

for all coarsenings $w$ of $v$ and $w^{\prime}$ of $w$.

Note that the set $\mathcal{X}$ is not uniquely determined. However, we will fix it throughout this paper and call the elements of $\mathcal{X}$ the monomials of $L E_{\mathcal{F}}(x)$. Correspondingly, we fix the residue fields $L E_{\mathcal{F}}(x) w=$ $\mathbb{R}\left(\mathcal{X}_{w}\right)^{\mathrm{h} \mathcal{F}}$ for all convex valuations $w$ on $L E_{\mathcal{F}}(x) w$.

Proof. According to [7, Theorem 6.30], $L E_{\mathcal{F}}(x)$ is of the form

$$
\mathbb{R}\left(x_{i} \mid i \in I\right)^{\mathrm{r} \mathcal{F}} \text { with } x_{i}>0 \text { and } v x_{i} \text { rationally independent, }
$$

and $x$ and $\log _{m} x, m \in \mathbb{N}$, among the $x_{i}$. Applying Lemma 3.1 with $w=v$, we find that the multiplicative group of $L E_{\mathcal{F}}(x)$ contains the divisible hull $\mathcal{X}$ of the subgroup generated by the $x_{i}$, and that $\mathcal{X}$ is the image of a cross-section. With $I_{v}=\emptyset$, we further obtain that $\mathbb{R}(\mathcal{X})^{\mathrm{r} \mathcal{F}}=\mathbb{R}(\mathcal{X})^{\mathrm{h} \mathcal{F}}$, which implies equation (7).

It remains to prove part b). Take a convex valuation $w$ on $L E_{\mathcal{F}}(x)$. The group $\mathcal{X}$ is isomorphic to the divisible value group $v L E_{\mathcal{F}}(x)$, so it is a $\mathbb{Q}$-vector space. For $\mathcal{X}_{w}=\left\{x^{\prime} \in \mathcal{X} \mid w x^{\prime}=0\right\}$, the values $v \mathcal{X}_{w}$ form a convex subgroup of this value group, which consequently is also divisible and a $\mathbb{Q}$-vector space. Hence also $\mathcal{X}_{w}$ is a $\mathbb{Q}$-vector space. We choose a basis $\mathcal{B}_{w}$ of $\mathcal{X}_{w}$ and a basis $\mathcal{B}_{w}^{\prime}$ of a complement of $\mathcal{X}_{w}$ in $\mathcal{X}$. We write $\mathcal{B}_{w}=\left\{x_{i} \mid i \in I_{w}\right\}, \mathcal{B}_{w}^{\prime}=\left\{x_{i} \mid i \in I_{w}^{\prime}\right\}$ and set $I=I_{w} \cup I_{w}^{\prime}$. As $\left\{x_{i} \mid i \in I\right\}$ is a basis of $\mathcal{X}$ which is isormorphic to the value group through the valuation, the values $v x_{i}, i \in I$, are rationally independent. Further, the elements $x_{i}, i \in I \backslash I_{w}=I_{w}^{\prime}$ are $\mathbb{Q}$-linearly independent over the $\mathbb{Q}$-vector space $\mathcal{X}_{w}$, which means that no nontrivial linear combination of these elements has value 0 under $w$. In other words, the values $w x_{i}, i \in I \backslash I_{w}$, are rationally independent.

Now we apply Lemma 3.1 to obtain that $\mathbb{R}\left(x_{i} \mid i \in I_{w}\right)^{\mathrm{r} \mathcal{F}}$ is a field of representatives for the residue field $\mathbb{R}\left(x_{i} \mid i \in I\right)^{\mathrm{r} \mathcal{F}} w$. We apply Lemma 3.1 again, this time to the field $\mathbb{R}\left(x_{i} \mid i \in I_{w}\right)^{\mathrm{r} \mathcal{F}}$ with its natural valuation $v$, to find that

$$
\mathbb{R}\left(x_{i} \mid i \in I_{w}\right)^{\mathrm{r} \mathcal{F}}=\mathbb{R}\left(\mathcal{X}_{w}\right)^{\mathrm{r} \mathcal{F}}=\mathbb{R}\left(\mathcal{X}_{w}\right)^{\mathrm{h} \mathcal{F}} .
$$

For coarsenings $w$ of $v$ and $w^{\prime}$ of $w$ we have that $w a=0$ implies $w^{\prime} a=0$, whence $\mathcal{X}_{w} \subseteq \mathcal{X}_{w^{\prime}}$. This yields eqation (9) and concludes the proof. 


\section{AN INTRINSIC VERSiON OF "TRUNCATION AT 0"}

Theorem 4.1. Take $h \in L E_{\mathcal{F}}(x)$ such that $v h<0$. Then there are convex valuations $w_{1}<w_{2}<\ldots<w_{k}=v$ on $L E_{\mathcal{F}}(x), m_{i} \in \mathbb{N}$, monomials $d_{i, j} \in \mathcal{X}$ and elements $c_{i, j} \in L E_{\mathcal{F}}(x) w_{i}, 1 \leq i \leq k, 1 \leq j \leq$ $m_{i}$, some $r_{h} \in \mathbb{R}$, and $h^{+} \in L E_{\mathcal{F}}(x)$ of value $v h^{+}>0$, such that

$h=c_{1,1} d_{1,1}+\ldots+c_{1, m_{1}} d_{1, m_{1}}+\ldots+c_{k, 1} d_{k, 1}+\ldots+c_{k, m_{k}} d_{k, m_{k}}+r_{h}+h^{+}$

with:

1) the values of the summands under the valuation $v$ are strictly increasing,

2) for each $i, 1 \leq i \leq k$,

$$
w_{i} c_{i, 1} d_{i, 1}<\ldots<w_{i} c_{i, m_{i}} d_{i, m_{i}}
$$

and the values $v d_{i, j}, 1 \leq j \leq m_{i}$ generate an archimedean ordered subgroup of $v L E_{\mathcal{F}}(x)$,

3) for each $i, 1 \leq i \leq k-1$,

$$
c_{i+1,1} d_{i+1,1}+\ldots+c_{i+1, m_{i+1}} d_{i+1, m_{i+1}}+\ldots+c_{k, 1} d_{k, 1}+\ldots+c_{k, m_{k}} d_{k, m_{k}}
$$

lies in $L E_{\mathcal{F}}(x) w_{i}$.

With these properties, the summands $c_{i, j}, d_{i, j}$ and the elements $r_{h}$ and $h^{+}$are uniquely determined.

Given the representation (11) of an element $h$ according to this theorem, the finite sum

$$
\operatorname{pp}(h):=c_{1,1} d_{1,1}+\ldots+c_{1, m_{1}} d_{1, m_{1}}+\ldots+c_{k, 1} d_{k, 1}+\ldots+c_{k, m_{k}} d_{k, m_{k}}
$$

will be called the principal part of $h$; we set $\operatorname{pp}(h):=0$ if $v h \geq 0$. The principal part is uniquely determined once the set of monomials in $L E_{\mathcal{F}}(x)$ is fixed. Note that $v\left(h-\operatorname{pp}(h)-r_{h}\right)>0$ with $r_{h} \in \mathbb{R}$.

The following lemma is the core of our proof:

Lemma 4.2. Let $(K, w)$ be a valued field with archimedean value group. Assume that $K=K_{0}\left(z_{j} \mid j \in J\right)$, where the values $w z_{j}, j \in J$, are rationally independent and $w$ is trivial on $K_{0}$. Denote by $\mathcal{Z}$ the multiplicative group $\left\langle z_{j} \mid j \in J\right\rangle$ generated by the elements $z_{j}$. Then the group ring

$$
R:=K_{0}[\mathcal{Z}]
$$

lies dense in $K$ (with respect to the topology induced by $w$ ). Moreover, for each $a \in K \backslash \mathcal{O}_{w}$ there are uniquely determined elements $c_{i} \in K_{0}$ 
and $d_{i} \in \mathcal{Z}$ with $w c_{i} d_{i}<0,1 \leq i \leq m$, such that

$$
a-\sum_{i=1}^{m} c_{i} d_{i} \in \mathcal{O}_{w} .
$$

The same holds if we replace $K$ by its henselization or its completion.

Proof. In order to prove that $R$ lies dense in $K$ we have to show that for every $a \in K$ and every $\alpha \in w K$ there is $a^{\prime} \in R$ such that $w\left(a-a^{\prime}\right)>\alpha$. Every $a \in K$ can be written as a quotient of two polynomials over $K_{0}$ in finitely many of the $z_{j}$, that is,

$$
a=\frac{b_{1}^{\prime} d_{1}^{\prime}+\ldots+b_{k}^{\prime} d_{k}^{\prime}}{b_{1}^{\prime \prime} d_{1}^{\prime \prime}+\ldots+b_{\ell}^{\prime \prime} d_{\ell}^{\prime \prime}}
$$

where $d_{1}^{\prime}, \ldots, d_{k}^{\prime} \in \mathcal{Z}$ are distinct, $d_{1}^{\prime \prime}, \ldots, d_{\ell}^{\prime \prime} \in \mathcal{Z}$ are distinct, and $b_{i}^{\prime}, b_{i}^{\prime \prime} \in K_{0} \backslash\{0\}$. From the rational independence of the values $w z_{j}$ it follows that every two distinct elements in $\mathcal{Z}$ and hence all $b_{i}^{\prime \prime} d_{i}^{\prime \prime}$ have distinct values. Therefore, we may assume that $b_{1}^{\prime \prime} d_{1}^{\prime \prime}$ is the unique summand of least value in the denominator. We write

$$
b_{1}^{\prime \prime} d_{1}^{\prime \prime}+\ldots+b_{\ell}^{\prime \prime} d_{\ell}^{\prime \prime}=b_{1}^{\prime \prime} d_{1}^{\prime \prime}(1-d) \quad \text { with } d:=-\frac{b_{2}^{\prime \prime} d_{2}^{\prime \prime}}{b_{1}^{\prime \prime} d_{1}^{\prime \prime}}-\ldots-\frac{b_{\ell}^{\prime \prime} d_{\ell}^{\prime \prime}}{b_{1}^{\prime \prime} d_{1}^{\prime \prime}} .
$$

Note that $\frac{d_{2}^{\prime \prime}}{d_{1}^{\prime \prime}}, \ldots, \frac{d_{\ell}^{\prime \prime}}{d_{1}^{\prime \prime}}$ are elements of $\mathcal{Z}$ of positive value. Hence, also $w d>0$, and $w(1-d)=0$. It follows that

$$
w\left(\frac{1}{1-d}-\sum_{i=0}^{\ell} d^{i}\right)=w\left(1-(1-d) \sum_{i=0}^{\ell} d^{i}\right)=w\left(-d^{\ell+1}\right)=(\ell+1) w d
$$

for every integer $\ell \geq 1$. Take $\alpha \in w K$. Since $w K$ is archimedean, we can choose $\ell$ as big as to obtain that

$$
(\ell+1) w d \geq \alpha-w\left(b_{1}^{\prime} d_{1}^{\prime}+\ldots+b_{k}^{\prime} d_{k}^{\prime}\right)\left(b_{1}^{\prime \prime} d_{1}^{\prime \prime}\right)^{-1} .
$$

For

$$
a^{\prime}:=\left(\frac{b_{1}^{\prime} d_{1}^{\prime}}{b_{1}^{\prime \prime} d_{1}^{\prime \prime}}+\ldots+\frac{b_{k}^{\prime} d_{k}^{\prime}}{b_{1}^{\prime \prime} d_{1}^{\prime \prime}}\right) \sum_{i=0}^{\ell} d^{i} \in R,
$$

this yields that

$$
w\left(a-a^{\prime}\right)=w\left(b_{1}^{\prime} d_{1}^{\prime}+\ldots+b_{k}^{\prime} d_{k}^{\prime}\right)\left(b_{1}^{\prime \prime} d_{1}^{\prime \prime}\right)^{-1}\left(\frac{1}{1-d}-\sum_{i=0}^{\ell} d^{i}\right) \geq \alpha,
$$

showing that $R$ lies dense in $K$. Deleting all summands from $a^{\prime}$ of value at least $\alpha$, we obtain a sum as in (12) such that $w c_{i} d_{i}<\alpha$ for all $i$ and $w\left(a-\sum_{i=1}^{n} c_{i} d_{i}\right) \geq \alpha$. For $\alpha=0$ this proves the existence of the 
elements $c_{i}, d_{i}$ as in the statement of the lemma. We have to prove their uniqueness.

Take two elements $r, r^{\prime} \in R$ in which all summands have value smaller than $\alpha$, and such that $w(a-r) \geq \alpha$ and $w\left(a-r^{\prime}\right) \geq \alpha$. It follows that $w\left(r-r^{\prime}\right) \geq \alpha$. Allowing the coefficients $b_{i}, c_{i}$ to be zero, we can write $r=c_{1} d_{1}+\ldots+c_{m} d_{m}$ and $r^{\prime}=b_{1} d_{1}+\ldots+b_{m} d_{m}$ where $d_{1}, \ldots, d_{m} \in \mathcal{Z}$ are distinct and $b_{i}, c_{i} \in K_{0}$. Then

$$
r^{\prime}-r=\left(b_{1}-c_{1}\right) d_{1}+\ldots+\left(b_{m}-c_{m}\right) d_{m} .
$$

As the value of this sum is equal to the minimum of its summands $\left(b_{i}-c_{i}\right) d_{i}$, we see that $w\left(b_{i}-c_{i}\right) d_{i} \geq w\left(r^{\prime}-r\right) \geq \alpha$ for all $i$. But if there is some $i$ such that $b_{i} \neq c_{i}$, then this yields $w d_{i} \geq \alpha$. As $b_{i} \neq 0$ or $c_{i} \neq 0$ it then follows that $w b_{i} d_{i}=w d_{i} \geq \alpha$ or $w c_{i} d_{i}=$ $w d_{i} \geq \alpha$, a contradiction to our initial assumption. Consequently, the representation $r=c_{1} d_{1}+\ldots+c_{m} d_{m}$ is uniquely determined when all $c_{i}$ are nonzero.

Every valued field is dense in its completion (by definition). Since $w K$ is archimedean, the henselization of $(K, w)$ lies in the completion and thus, $(K, w)$ is also dense in its henselization. Since density is transitive, we find that $R$ is also dense in the henselization and in the completion of $(K, w)$. It follows that the assertions we have proved for $a \in K$ also hold when $a$ lies in the henselization or completion.

The following is [7, Lemma 6.41]:

Lemma 4.3. Let $x_{i} \in M$ such that $x_{i}>0$ and the values $v x_{i}, i \in I$ are rationally independent. Then

$$
\mathbb{R}\left(x_{i} \mid i \in I\right)^{\mathrm{r} \mathcal{F}}=\bigcup_{I_{0} \subset I \text { finite }} \bigcup_{k \in \mathbb{N}} \mathbb{R}\left(x_{i}^{1 / k} \mid i \in I_{0}\right)^{\mathrm{h} \mathcal{F}} .
$$

In order to prove Theorem 4.1, take any $h \in L E_{\mathcal{F}}(x)$. We will work with the representation of $L E_{\mathcal{F}}(x)$ as given in Theorem 3.2. Lemma 4.3 shows that there is a finitely generated subgroup $\mathcal{X}_{h}$ of $\mathcal{X}$ such that $h \in \mathbb{R}\left(\mathcal{X}_{h}\right)^{\mathrm{h} \mathcal{F}} \subset \mathbb{R}\left(\mathcal{X}_{h}\right)^{\mathrm{r} \mathcal{F}}$. Denote by $\mathcal{X}^{\prime}$ the divisible hull of $\mathcal{X}_{h}$ inside the divisible group $\mathcal{X}$. Since $v \mathcal{X}^{\prime}$ is isomorphic to $\mathcal{X}^{\prime}$ which is the divisible hull of a finitely generated abelian group, it must have finite rational rank $\operatorname{dim}_{\mathbb{Q}} \mathbb{Q} \otimes v \mathcal{X}^{\prime}$. Therefore, $v \mathcal{X}^{\prime}$ has only finitely many convex subgroups, say,

$$
v \mathcal{X}^{\prime}=\Gamma_{1} \supset \Gamma_{2} \supset \ldots \supset \Gamma_{k+1}=\{0\}
$$

such that $\Gamma_{i} / \Gamma_{i+1}$ is archimedean ordered, for $1 \leq i \leq k$. Further, we choose convex valuations $w_{1}<\ldots<w_{k}$ on $L E_{\mathcal{F}}(x)$ such that 
the restriction of $w_{i}$ to $K$ is a convex valuation corresponding to $\Gamma_{i+1}$, having value group $v \mathcal{X}^{\prime} / \Gamma_{i+1}$. Since $\Gamma_{k+1}=\{0\}$, we can choose $w_{k}=v$. Each

$$
\mathcal{X}_{i}^{\prime}:=\left\{x^{\prime} \in \mathcal{X}^{\prime} \mid v x^{\prime} \in \Gamma_{i}\right\}, \quad 1 \leq i \leq k
$$

is a $\mathbb{Q}$-sub vector space of the $\mathbb{Q}$-vector space $\mathcal{X}^{\prime}$. We choose a $\mathbb{Q}$-basis $\mathcal{B}_{k}$ of $\mathcal{X}_{k}^{\prime}$, and if $k>1, \mathbb{Q}$-bases $\mathcal{B}_{i}$ of complements of $\mathcal{X}_{i+1}^{\prime}$ in $\mathcal{X}_{i}^{\prime}$ for $1 \leq i \leq k-1$. We obtain that $\mathcal{B}:=\bigcup_{i} \mathcal{B}_{i}$ is a $\mathbb{Q}$-basis of $\mathcal{X}^{\prime}$ and that $h \in \mathbb{R}(\mathcal{B})^{\mathrm{r} \mathcal{F}}$.

From Lemma 4.3 we infer that $h \in \mathbb{R}\left(b^{1 / \ell} \mid b \in \mathcal{B}\right)^{\mathrm{h} \mathcal{F}}=: K$ for some $\ell \in \mathbb{N}$. Since we may replace each basis element $b$ by $b^{1 / \ell}$, we can assume that $\ell=1$.

Now we proceed by induction on $k$. We assume that $k=1$ or that the theorem has been proven for all elements in $\mathbb{R}(\overline{\mathcal{B}})^{\mathrm{h} \mathcal{F}}$, where $\overline{\mathcal{B}} \subset \mathcal{B}$ corresponds to a value group that has less convex subgroups than $v \mathcal{X}^{\prime}$.

The value group of the convex valuation $w_{1}$ on $K$ is the archimedean ordered group $v \mathcal{X}^{\prime} / \Gamma_{k-1}$. We set $\overline{\mathcal{B}}=\emptyset$ if $k=1$, and $\overline{\mathcal{B}}=\bigcup_{2 \leq i \leq k} \mathcal{B}_{i}$ if $k>1$. From Lemma 3.1 we infer that $\mathbb{R}(\overline{\mathcal{B}})^{\mathrm{h} \mathcal{F}}$ is a field of representatives for the residue field $K w_{1}$.

We apply Lemma 4.2 with $\mathcal{Z}$ equal to the group generated by $\mathcal{B}_{1}$ and $K_{0}=\mathbb{R}(\overline{\mathcal{B}})^{\mathrm{h} \mathcal{F}}$ to deduce the existence of uniquely determined elements

$$
c_{1, j} \in \mathbb{R}(\overline{\mathcal{B}})^{\mathrm{h} \mathcal{F}} \subset \mathbb{R}\left(\mathcal{X}_{w_{1}}\right)^{\mathrm{h} \mathcal{F}}=L E_{\mathcal{F}}(x) w_{1}
$$

and

$$
d_{1, j} \in \mathcal{Z} \subset \mathcal{X}, \quad 1 \leq j \leq m_{1}
$$

with

$$
w_{1} c_{1,1} d_{1,1}<\ldots<w_{i} c_{1, m_{1}} d_{1, m_{1}}<0
$$

and such that $w_{1}\left(h-\sum_{j=1}^{m_{1}} c_{1, j} d_{1, j}\right) \geq 0$. Thus, there is a unique element $\bar{h} \in \mathbb{R}(\overline{\mathcal{B}})^{\mathrm{h} \mathcal{F}}$ such that

$$
w_{1}\left(h-\sum_{i=1}^{m} c_{i} d_{i}-\bar{h}\right)>0 .
$$

By definition of $\mathcal{B}_{1}$ we have that $v \mathcal{Z} \subseteq \Gamma_{1}$ and $v \mathcal{Z} \cap \Gamma_{2}=\{0\}$, which shows that $v \mathcal{Z}$ is archimedean. The same consequently holds for its subgroup that is generated by the values $v d_{1, j}, 1 \leq j \leq m_{1}$.

If $k=1$, then $\mathbb{R}(\overline{\mathcal{B}})^{\mathrm{h} \mathcal{F}}=\mathbb{R}$ and we can set $r_{h}=\bar{h} \in \mathbb{R}$ to obtain that $v\left(h-\sum_{i=1}^{m} c_{i} d_{i}-r_{h}\right)>0$.

If $k>1$, then by induction hypothesis we know that our theorem holds for the element $\bar{h}$. We can thus write $\bar{h}=c_{2,1} d_{2,1}+\ldots+c_{2, m_{2}} d_{2, m_{2}}+\ldots+c_{k, 1} d_{k, 1}+\ldots+c_{k, m_{k}} d_{k, m_{k}}+r_{\bar{h}}+\bar{h}^{+}$ 
such that the conditions of Theorem 4.1 are satisfied for $\bar{h}$ in place of $h$ (and with $w_{1}$ omitted). Now we set $r_{h}:=r_{\bar{h}}$ and $h^{+}:=\bar{h}^{+}$to obtain a representation of the form (11) for $h$. It is straightforward to see that properties 2) and 3) are satisfied. Also 1) holds since $w_{1} z \neq 0$ for all $z \in \mathcal{Z}$, which implies that $v c_{1, j} d_{1, j}<\Gamma_{2}$ for $1 \leq j \leq m_{1}$, whereas $w_{1} y=0$ for all $y \in \mathbb{R}(\overline{\mathcal{B}})^{\mathrm{h} \mathcal{F}}$, which implies that $v c_{i, j} d_{i, j} \in \Gamma_{2}$ for $2 \leq i \leq k$ and $1 \leq j \leq m_{i}$. This completes the proof of Theorem 4.1 .

\section{Applications}

Theorem 5.1. Let $f, g: \mathbb{R} \rightarrow \mathbb{R}$ be ultimately positive $\mathbb{R}$-definable functions. Then $f$ is asymptotic to $r g$ on $\mathbb{R}$ for some positive $r \in \mathbb{R}$ if and only if the germs $\log f$ and $\log g$ in $H(\mathbb{R})$ have the same principal part.

Proof. We know from (5) that $f$ is asymptotic to $r g$ on $\mathbb{R}$ if and only if $v(\log f-\log r g)>0$. This in turn is equivalent to $v(\log f-\log g) \geq 0$, since if the latter holds, then there is some $r_{0} \in \mathbb{R}$ such that $v(\log f-$ $\left.\log g-r_{0}\right)>0$, and we set $r=\exp r_{0}$. By the uniqueness of the principal part, $v(\log f-\log g) \geq 0$ if and only if $\operatorname{pp}(\log f)=\operatorname{pp}(\log g)$.

To apply this theorem in the spirit of the Hardy problem, we take $\mathcal{F}$ to be any set of restricted analytic functions, closed under partial derivations. Then by applying [7, Theorem 6.30] simultaneously for $\mathcal{F}$ and $\mathcal{F}_{\text {an }}$, we find index sets $I_{\mathcal{F}} \subset I$ and elements $x_{i}$ such that $L E_{\mathcal{F}}(x)=\mathbb{R}\left(x_{i} \mid i \in I_{\mathcal{F}}\right)^{\mathrm{r} \mathcal{F}}$ and $L E_{\mathcal{F}_{\text {an }}}(x)=\mathbb{R}\left(x_{i} \mid i \in I\right)^{\mathrm{r} \mathcal{F}_{\text {an }}}$. So the monomials of $L E_{\mathcal{F}}(x)$ will also be monomials of $L E_{\mathcal{F}_{\text {an }}}(x)$. Moreover, we can take

$$
L E_{\mathcal{F}}(x) w \subseteq L E_{\mathcal{F}_{\text {an }}}(x) w
$$

for each convex valuation $w$ and suitable $m_{0}$, according to Theorem 3.2 . Using principal parts determined by this choice of the $x_{i}$ and the residue fields, we get:

Corollary 5.2. Assume that $h: \mathbb{R} \rightarrow \mathbb{R}$ is definable in $\mathbb{R}_{\text {an,exp. Then }}$ $\exp h$ is asymptotic to a composition of semialgebraic functions, exp, log and restricted analytic functions in $\mathcal{F}$, if and only if $\operatorname{pp}(h) \in L E_{\mathcal{F}}(x)$.

As an example, let us reconsider the Hardy problem. Here we assume in addition that the $x_{i}$ include $x$ (cf. Theorem 3.2).

Take two functions $f, g: \mathbb{R} \rightarrow \mathbb{R}$, definable in $\mathbb{R}_{\text {an,exp }}$. Assume that $\exp f(x)$ is asymptotic to $g(x)$, that is, $\lim _{x \rightarrow \infty} \frac{\exp f(x)}{g(x)}=1$. This is equivalent to $\lim _{x \rightarrow \infty} f(x)-h(x)=0$, where $h:(r, \infty) \rightarrow \mathbb{R}$ for suitable $r \in \mathbb{R}$ is the function $\log g(x)$, which again is definable in $\mathbb{R}_{\mathrm{an} \text {,exp }}$. This 
means that the function $f(x)-h(x)$ is ultimately smaller than every nonzero constant function. Equivalently, its germ $f-h$ in $H\left(\mathbb{R}_{\text {an,exp }}\right)$ is infinitesimal, or in other words, $v(f-h)>0$.

As in [1, let the function $i(x)$ denote the compositional inverse of the function $x \log x$. Identifying $i(x)$ with its germ, we have that $i(x) \in$ $H\left(\mathbb{R}_{\text {an,exp }}\right)$. But by an argument about Liouville extensions of the Hardy field $\mathbb{R}(x),\left[1\right.$, Corollary 4.6] shows that $i(x) \notin L E:=L E_{\mathcal{F}_{\text {an }}}(x)$. Assume that $\exp i(x)$ were asymptotic to a function $g(x)$ which is a composition of semialgebraic functions, exp and log. Through identification with its germ, the latter means that $g(x) \in L E$. Then also $h(x):=\log g(x) \in L E$, and $v(i(x)-h(x))>0$. Further, one shows as in [1] that there is a convergent power series $f(X, Y)$ such that

$$
i(x)=\frac{x}{\log x}\left(1+f\left(\frac{\log \log x}{\log x}, \frac{1}{\log x}\right)\right) .
$$

Now let $w$ be the convex valuation corresponding to the largest convex subgroup not containing $v x$. This contains $v \log x$. Therefore, $w \log x=$ 0 and $w \frac{\log x}{x}=-w x>0$. With

$$
\tilde{f}:=f\left(\frac{\log \log x}{\log x}, \frac{1}{\log x}\right) \in \mathbb{R}(\log x, \log \log x)^{r \mathcal{F}_{\text {an }}} \subseteq L E_{\mathcal{F}_{\text {an }}}(\log x) w,
$$

the representation of $i(x)$ is just $i(x)=c x$, where $c=\frac{1}{\log x}(1+\tilde{f}) \in$ $H\left(\mathbb{R}_{\text {an,exp }}\right) w$. Thus, $\operatorname{pp}(i(x))=i(x) \notin L E$. Hence by our Corollary 5.2. $\exp i(x)$ is not asymptotic to any element of $L E$.

Let us give a further application of Theorem 4.1. Denote by $\mathcal{L}_{\mathcal{F}}$ the language of ordered rings, enriched by symbols for the functions from $\mathcal{F}$. Recall that every generalized power series field $\mathbb{R}((G))$ has a canonical cross-section, sending $\alpha \in G$ to the element $1_{\alpha} \in \mathbb{R}((G))$ which has a 1 at $\alpha$ and zeros everywhere else. $\left(1_{\alpha}\right.$ is the characteristic function of the singleton $\{\alpha\}$.)

Corollary 5.3. Take any $\mathcal{L}_{\mathcal{F}}$-embedding of $L E_{\mathcal{F}}(x)$ in some generalized power series field $\mathbb{R}((G))$, and denote by $L$ its image in $\mathbb{R}((G))$. Assume that the restriction of the canonical cross-section of $\mathbb{R}((G))$ to $v L$ is a cross-section $\pi$ of $(L, v)$, and that $L=\mathbb{R}(\pi v L)^{\mathrm{r} \mathcal{F}}$. Then the nonzero elements of the support of each element in $L$ are bounded away from 0 .

Proof. For every convex valuation $w$ with associated convex subgroup $H_{w} \subset G$, we have that $\mathbb{R}((G)) w=\mathbb{R}\left(\left(H_{w}\right)\right)$.

Let $I \subset v L$ be a maximal set of rationally independent values. Set $x_{i}:=1_{\alpha}$ for $i=\alpha \in I$. Then $\mathbb{R}\left(x_{i} \mid i \in I\right)^{\mathrm{r}}=\mathbb{R}(\pi v L)^{\mathrm{r}}$ and hence, 
$\mathbb{R}\left(x_{i} \mid i \in I\right)^{\mathrm{r} \mathcal{F}}=\mathbb{R}(\pi v L)^{\mathrm{r} \mathcal{F}}=L$ by hypothesis. The monomials obtained from the $x_{i}$ are precisely the elements of the form $r \cdot 1_{\alpha}$ with $r \in \mathbb{R}$ and $\alpha \in v L$. Note that if $\alpha<H_{w}$, then for every $c \in \mathbb{R}\left(\left(H_{w}\right)\right)$, the support of $\operatorname{cr} 1_{\alpha}$ is bounded away from 0 by every element $\beta$ which satisfies $\alpha+H_{w}<\beta<0$. For example, $\beta=\alpha / 2$ is a good choice.

Take $h \in L$ and consider the representation (11) with respect to the monomials $x_{i}$ and the residue fields $\mathbb{R}\left(\left(H_{w}\right)\right)$. Now support $(h) \backslash\{0\}$ is the union of the support of $c_{1} d_{1}+\ldots+c_{m} d_{m}$ and the support of $h^{+}$. The latter is bounded away from 0 by $v h^{+}$. The support of $c_{1} d_{1}+\ldots+c_{m} d_{m}$ is the union of the supports of $c_{1} d_{1}, \ldots, c_{m} d_{m}$. This union is bounded away from 0 by $\frac{1}{2} v d_{m}$.

Note that the embeddings of $H\left(\mathbb{R}_{a n, \exp }\right)$ and of $L E$ in the logarithmic power series field $\mathbb{R}((t))^{L E}$ given in [1] satisfy the conditions of the corollary. (Recall that $\mathbb{R}((t))^{L E}$ can be viewed as a subfield of a suitable power series field.)

\section{REFERENCES}

[1] van den Dries, L. - Macintyre, A. - Marker, D.: Logarithmic-Exponential Power Series, J. London Math. Soc. 56 (1997), 417-434

[2] van den Dries, L. - Speissegger, P.: The field of reals with multisummable series and the exponential function, Proc. London Math. Soc. 81 (2000), 513565

[3] Engler, A.J. - Prestel, A. : Valued fields, Springer Monographs in Mathematics. Springer-Verlag, Berlin, 2005

[4] Kuhlmann, F.-V. - Kuhlmann, S. : Residue fields of arbitrary convex valuations on restricted analytic fields with exponentiation I $\&$ II, The Fields Institute Preprint Series (1996)

[5] Kuhlmann, F.-V. - Kuhlmann, S.: Valuation theory of exponential Hardy fields II, preprint (2000), arXiv 1206.0711

[6] Kuhlmann, F.-V. - Kuhlmann, S. : Valuation theory of exponential Hardy fields I, Math. Zeit., 243, (2003), 671-688

[7] Kuhlmann, S.: Ordered Exponential Fields, The Fields Institute Monographs 12, AMS Publications (January 2000)

[8] Ribenboim, P.: Théorie des valuations, Les Presses de l'Université de Montréal, Montréal, 1st ed. (1964), 2nd ed. (1968)

[9] Wilkie, A. : Complex continuations of $\mathbb{R}_{\text {an,exp-definable unary functions with }}$ a diophantine application, J. London Math. Soc. first published online March 8, (2016), doi:10.1112/jlms/jdw007

Institute of Mathematics, University of Silesia, Ul. Bankowa 14, 40-007 Katowice, Poland

E-mail address: fvk@math.us.edu.pl 
FB Mathematik und Statistik, Universität Konstanz, 78464 KonSTANZ, Germany

E-mail address: salma.kuhlmann@uni-konstanz.de 